Radiologe 2011 · 51:653

DOI 10.1007/s00117-011-2217-8

Online publiziert: 30 . Juli 2011

(c) Springer-Verlag 2011

\author{
M. Karul \\ Klinik für Radiologie und Nuklearmedizin, \\ Universitätsklinikum Schleswig-Holstein, Campus Lübeck, Lübeck
}

\title{
RECIST ist einfach - mit RECIST zu arbeiten jedoch nicht!
}

cer veröffentlicht wurde. Die RECISTVersion 1.1 basiert auf den Erkenntnissen aus einer Datenbank mit ca. 6500 Patienten und ca. 18.0oo Läsionen.

Nishino et al. vergleichen die RECISTVersion 1.1 mit der Originalarbeit 1.0 und begutachten die umfassenden Änderungen:

- Reduktion der Anzahl der Zielläsionen,

- Beurteilung einzelner Lymphknoten,

- Überarbeitung der Kategorie „progressive disease",

- Berücksichtigung einer eindeutigen Zunahme der Nichtzielläsionen,

- Kriterien zur Identifikation neuer Läsionen mittels ${ }^{18} \mathrm{~F}$-Fluordesoxyglukose-Positronenemissionstomographie (FDG-PET).

Die ausschließliche 1-D-Messung des längsten Durchmessers ist nach Meinung der Autoren ungenügend. Sie schlagen vor, das Therapiemonitoring z. B. mit der hochauflösenden Mehrschichtcomputertomographie (MSCT) unter Anwendung einer softwarebasierten Volumenmessung durchzuführen. Eine vergleichende 3-DVolumenmessung für kleine Größenveränderungen einer Läsion sei definitiv sensitiver als eine 1-D-Vermessung des längsten Durchmessers.

Die Leitlinie ist alles in allem sicher nicht perfekt und bietet dem kritischen Leser jede Menge Angriffspunkte. Bei unterschiedlicher Anwendung entstehen z. B. häufig Messungenauigkeiten, die sich dann als systematische Fehler durch das gesamte Follow-up ziehen können. RECIST erfordert daher eine konstante, ge- wissenhafte und konsequente Anwendung der Kriterien.

Eine Besonderheit der Neuroblastome im frühen Säuglingsalter ist, dass sich die Tumoren im Stadium 4S (Metastasen „nur“ in Leber, Haut und Knochenmark) nach einem Progress spontan zurückbilden können. Diese durchaus gute Prognoseabschätzung kann durch RECIST nicht berücksichtigt werden. Ganz zu schweigen von der hohen Strahlenbelastung bei einer CT sind die Tumorgrenzen entweder nicht eindeutig abgrenzbar oder differenzialdiagnostisch nicht von einem entzündlichen Prozess zu unterscheiden. Oft werden die Messungen auch durch Lymphknoten in der Nähe der Zielläsionen verfälscht.

Zusammenfassend untermauert die vorliegende Übersichtsarbeit zu Recht die Bedeutung der Leitlinie und arbeitet die wesentlichen Änderungen der Version 1.1 im Vergleich zur Originalversion 1.o heraus. Der Vorschlag, die Zielläsionen nicht mehr ein-, sondern dreidimensional zu messen, entspricht einem Paradigmenwechsel. Diese Zukunftsvision der Autoren kann nur befürwortet werden, wenn eine weit verbreitete Verfügbarkeit der erforderlichen Scanner ausreichende Standards gewährleistet.

\section{Korrespondenzadresse \\ M. Karul}

Klinik für Radiologie und Nuklearmedizin, Universitätsklinikum Schleswig-Holstein, Campus Lübeck,

Ratzeburger Allee 160, 23538 Lübeck

murat.karul@uk-sh.de

Interessenkonflikt. Der korrespondierende Autor gibt an, dass kein Interessenkonflikt besteht. vision der Leitlinie, die 2009 von Eisenhauer et al. im European Journal of Can- 